\title{
Investigating Auditory and Visual Memory and Sequencing - Exploring Strengthening Therapeutics for Alzheimer's Patients
}

\author{
Chloe Ganjian ${ }^{1}$ and Merideth McCarthy ${ }^{1}$ \\ ${ }^{1}$ North Shore Hebrew Academy High School, Great Neck, NY, USA
}

\begin{abstract}
$\underline{\text { ABSTRACT }}$
In modern society, the inherent differences found between females and males are a sensitive topic of debate. It is common for people to associate females with the possession of stronger and more profound visual and auditory memory in comparison to males. This research explored the variances between the sexes by examining and comparing the auditory and visual memories of a population of females and a population of males. The auditory and visual memories of the different sexes will be analyzed through a variety of tasks that will measure their memory capabilities. Participants of the experiment took two pre-designed tests, a different test for each type of memory. The auditory test included four distinct sounds that played in a specific pattern and challenged the participants' memory capacities. Through the use of MATLAB, a program developed by MathWorks, and by using coding language, a visual memory game was developed for the visual test. This test contained four different colors that illuminated in a specific sequence. Each participant completed two trials for each type of test to allow them the opportunity to achieve their maximum potential. The analysis of the results contradicted stereotypical beliefs embedded in societal norms. Males collectively demonstrated a higher capacity for memory than females. Notably, both sexes improved their memory performance on the visual tests more than the auditory tests. An application of these results could be used as therapeutic exercises to help strengthen the memory of patients who suffer from Alzheimer's disease and other memory loss.
\end{abstract}

\section{Introduction}

Auditory memory, also known as echoic memory, is one of the sensory memory registries. It is a type of short-term sensory memory. Echoic memory is a component that makes up the sensory memory and is in charge of the shortterm auditory information that is received from the environment (Nishihara et al., 2014). Diverse studies have noted that this system may store a greater amount of information for a longer period of time, 3-4 seconds longer on average, than visual memory (Nees, 2016). Auditory, or echoic memory, is made up of a storage area of large, naturally precategorized, limited persistent auditory information (Brady et al., 2011).

The auditory stimuli will automatically be sent to the central auditory processor, which is in charge of changing the electric signals from sounds into mental concepts, creating a type of sound image (Archakov et al., 2020). This image can be stored in the brain for a short period of time. The sound image can only be replayed during a short period of time following the auditory stimuli. Sensory memory is measured in the primary auditory cortex. This auditory memory storage area extends through different areas of the brain because it requires the use of diverse processes. The majority of these brain regions are located in the prefrontal cortex (PFC), which is also where the executive and attentional control are monitored (Plakke \& Romanski, 2014).

Based upon research findings related to neuroscience and brain plasticity, the more the neural circuit is utilized, the stronger the neural circuit gets. This suggests that the circuits used in auditory processing can be improved as well. In order to improve the auditory memory, the person needs to actively practice and exercise their brain (Ferguson \& Henshaw, 2015). 
Iconic memory is a short term visual memory that lasts only a few seconds before getting disregarded. For example, the brain uses iconic memory to remember an image that was observed. The sensory memory stores all of the information that was experienced through the five major senses consisting of touch, taste, sight, hearing and smell. This storage of the combined stimuli is an automatic response by the brain. If sufficient attention is given to this stimulus, the information may then pass into the short-term memory. At this point, that information can get encoded and passed into the long-term memory (Nishihara et al., 2014). However, in most cases, sensory memory is thrown out in just a matter of a few seconds. When an ambiguous sensory input enters the visual system, it goes into the iconic memory. The visual system includes iconic memory, visual short-term memory and long-term memory. Iconic memory is simply the brain's mechanism of processing visual information.

The occipital lobe is the central part of the brain involved in iconic memory. This lobe is responsible for processing and regulating visual information. When a person "sees" something, the visual information is received by the photoreceptor cells in the eyes and is then sent to the occipital lobe through electrical signals (Barton \& Brewer, 2013). At this point, it is stored for a few milliseconds before either being forgotten or transferred to the temporal lobe. This visual memory, or visual persistence, is then converted from visual short-term memory to long-term memory by the hippocampus, which is located inside the temporal lobe (Barton \& Brewer, 2013).

The iconic memory and echoic memory are the two most extensively studied sensory memories. One big difference between iconic memory and echoic memory is regarding the duration and capacity. While echoic memory lasts up to 2 seconds (Nees, 2016), iconic memory or short-term visual memory lasts roughly 300 - 500 milliseconds (Lu et al., 2005). However, when analyzing the ability to maintain a particular amount of subjects within the different types of memory, the average human in reference to iconic memory can preserve 3-4 items (Barton \& Brewer, 2013), whereas the capacity of the echoic memory can last up to 20 seconds (Nees, 2016). In the first stage, any incoming sensory or perceptual information is held in the sensory memory. The information gathered through the senses is an exact copy of what you perceive. This information is stored in the sensory memory for a very short duration. For instance, as soon as a person receives a visual stimulus, such as the vision of an apple, it is processed by the eyes and the information is moved into the occipital lobe. Next, recognition occurs, and this information is then placed into iconic memory. This entire process, from the beginning to the end, takes place extraordinarily quickly (Barton \& Brewer, 2013). From the iconic memory, only a limited amount of information that is consciously paid attention to moves into the visual working memory, this is the short-term memory for visual stimuli. Any remaining, unprocessed information is discarded (Friedman et al., 2018).

This short-term memory does not have unlimited capacity. It can only store a limited amount of information (Brady et al., 2011). Conscious perception makes a significant difference in the memory process. The information can remain in the working memory for several minutes before being discarded or stored in long-term memory (Brady et al., 2011). Memory exercises can be used to strengthen attention, which in turn will improve retention and recall (Friedman et al., 2018).

The purpose of this study is to test the echoic and iconic memory against the gender of the individual, to examine if males or females innately have a more acute memory capability. Specifically, the hypothesis for this experiment is that auditory memory is stronger than visual memory; furthermore, females have a stronger capability in both auditory and visual memory than males.

\section{Materials}

A total of 40 participants, 20 males and 20 females, voluntarily participated in this experiment. The experiment consisted of a visual test and an auditory test. There were two trials for each of these tests. To eliminate any experimental bias, the order in which the participants received the tests were random. This resulted in an equal number of participants performing the visual test first, as well as an equal number of those who first performed the auditory test first.

A coded visual memory game was developed for the visual test of this experiment. The game was coded using MATLAB, a program developed by MathWorks. Using coding language, the strategic development and format 
of the visual game were made possible. This game consisted of four different colors; red, blue, green and yellow. A pattern of colors would light up, and participants were asked to repeat the pattern and sequence of the colors that were illuminated. There were 14 rounds, and every round, a new color was added to the end of the pattern if the participant correctly repeated the pattern. The colors were programmed to illuminate in a specific sequence, which was held constant for all participants.

\section{Methods}

Participants were asked to press the colors in the sequence in which they were brightly displayed. When a participant correctly repeated the colors, a new color was added to the pattern, and then the participant was asked to repeat the whole process from the beginning. This procedure was repeated until the pattern was incorrectly repeated by the participant or until the participant correctly identified the patterns in all 14 rounds. The sequence of the colors was yellow, red, green, red, red, blue, yellow, green, blue, red, green, green, blue, and then blue. This sequence of colors was automatically generated by the program, and each participant was given the same pattern, to negate any personal meanings that may be particular for different participants. The purpose of keeping the pattern the same for the tests was to eliminate any participant bias. In this visual test, each participant was given two trials to replicate the ordered display of colors to the best of their abilities. The scores derived from each of the two trials were individually recorded and included in the participant's final score.

In the auditory test, a series of four sound clips were played: a police siren, a bell tone, office phone ringtone and thunder. Each of the sounds were played once for the participants before the test in order to ensure that they were familiar with the names of these four distinct sounds. Each of the sounds played corresponded to a specific color. The police siren was coupled with the color green. The thunder sound corresponded to the color red. The office phone ringtone corresponded to the color yellow. The bell tone corresponded to the color blue. As a result, the pattern of the sounds corresponded to the pattern of the colors displayed in the visual test. The pattern of the sounds played was in this order: office phone ringtone, thunder, police siren, thunder, thunder, bell tone, office phone ringtone, police siren, bell tone, thunder, police siren, police siren, bell tone, bell tone.

Similar to the visual test, the auditory test started off with one sound, and the participant was asked to identify the name of the sound. If the sound was correctly identified, another sound was added to the pattern, and the participant was asked to identify the whole pattern from the very first sound to the newest addition. This process continued on until a sound was incorrectly identified, or until the participant identified the correct sequence in all 14 rounds. In this auditory test, each participant was given two trials to try to remember the sounds to the best of their abilities. As in the visual test, the scores derived from each of the two trials of the auditory test were individually recorded and included in the participant's final score.

\section{Results}

After analysis of the collected data, it was evident that there was an improvement in the second trials of both the auditory and visual tests for both the female and male populations.

Table 1. Collected data from the experiment.

\begin{tabular}{|c|c|c|c|c|c|}
\hline Test Group & Test Type & $\begin{array}{c}\text { Trial Num- } \\
\text { ber }\end{array}$ & $\begin{array}{c}\text { Combined } \\
\text { Score Totals } \\
\text { of Partici- } \\
\text { pants }\end{array}$ & $\begin{array}{c}\text { Average } \\
\text { Score Out } \\
\text { of 14 }\end{array}$ & $\begin{array}{c}\text { Average } \\
\text { Score from } \\
\text { Trials Out } \\
\text { of 14 }\end{array}$ \\
\hline \multirow{2}{*}{$\begin{array}{c}\text { Female } \\
(\mathbf{n = 2 0})\end{array}$} & Auditory & 1 & 134 & 6.7 & 7.3 \\
\cline { 2 - 5 } & Auditory & 2 & 158 & 7.9 & \\
\hline
\end{tabular}




\begin{tabular}{|c|c|c|c|c|c|}
\hline & Visual & 1 & 165 & 8.3 & \multirow{2}{*}{9.2} \\
\cline { 2 - 5 } & Visual & 2 & 202 & 10.1 & \\
\hline \multirow{3}{*}{ Male (n=20) } & Auditory & 1 & 143 & 7.2 & \multirow{2}{*}{7.8} \\
\cline { 2 - 5 } & Auditory & 2 & 168 & 8.4 & \\
\cline { 2 - 5 } & Visual & 1 & 174 & 8.7 & \multirow{2}{*}{9.5} \\
\cline { 2 - 5 } & Visual & 2 & 203 & 10.2 & \\
\hline
\end{tabular}

For example, in the female visual tests, the average score improved by 1.8 points in the second trial. This produced a $13 \%$ increase in score when comparing the first trial to the second trial. A paired sample t-test was conducted, which yielded a value of $p=0.003$, providing statistical evidence that a significant difference does exist between the first and second trials. In the female auditory test, the average score improved by 1.2 points in the second trial. This produced an $8 \%$ increase in score when comparing the first trial to the second trial. A paired sample t-test was conducted, which yielded a value of $p=0.031$, providing statistical evidence that a significant difference does exist between the first and second trial.

Likewise, in the male visual tests, the average score improved by 1.5 points in the second trial. This produced an $11 \%$ increase in score when comparing the first trial to the second trial. A paired sample t-test was conducted, which yielded a value of $p=0.027$, providing statistical evidence that a significant difference does exist between the first and second trial. In the male auditory test, the average score improved by 1.2 points in the second trial. This produced a $9 \%$ increase in score when comparing the first trial to the second trial. A paired sample t-test was conducted, which yielded a value of $p=0.019$, providing statistical evidence that a significant difference does exist between the first and second trial.

Within the two types of memory assessed, the visual memory of the female population was significantly stronger than their auditory memory. Overall, females performed better in the visual tests than the auditory tests. The average score for the female visual test was 9.2 out of 14, and the average score for their auditory test was only 7.3 out of 14. Collectively, their achieved visual memory score was $14 \%$ higher than their achieved auditory memory score.

Similarly, the male participants also performed better in the visual tests than the auditory tests. The average score for the male visual tests was 9.5 out of 14 . Meanwhile, the average of their auditory tests was only 7.8 out of 14. Collectively, their achieved visual memory score was $12 \%$ higher than their achieved auditory memory score.

Overall, males performed at a higher level of accuracy than females in their ability to retain their memory within the different categories of the experiment. The average score of the male visual tests was 9.5 out of 14, whereas for females, it was only 9.2 out of 14 . The male population performed better than the female population in the auditory tests as well. The average score of the male auditory tests was 7.8 out of 14 , while for females, it was only 7.3 out of 14.

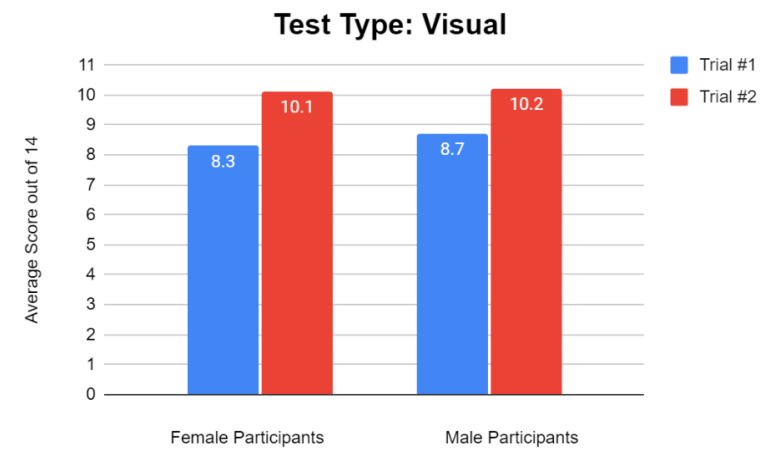

Figure 1. Average Visual Test Scores of Both Females and Males 


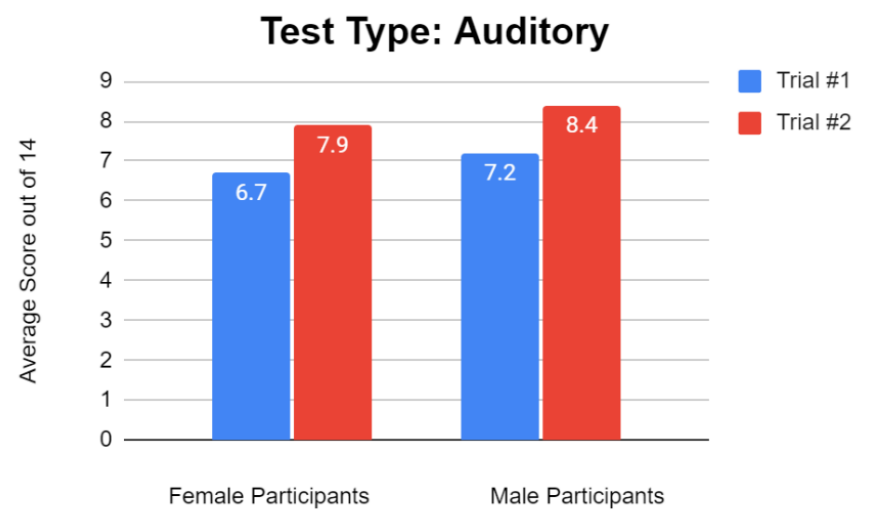

Figure 2. Average Auditory Test Scores of Both Females and Males

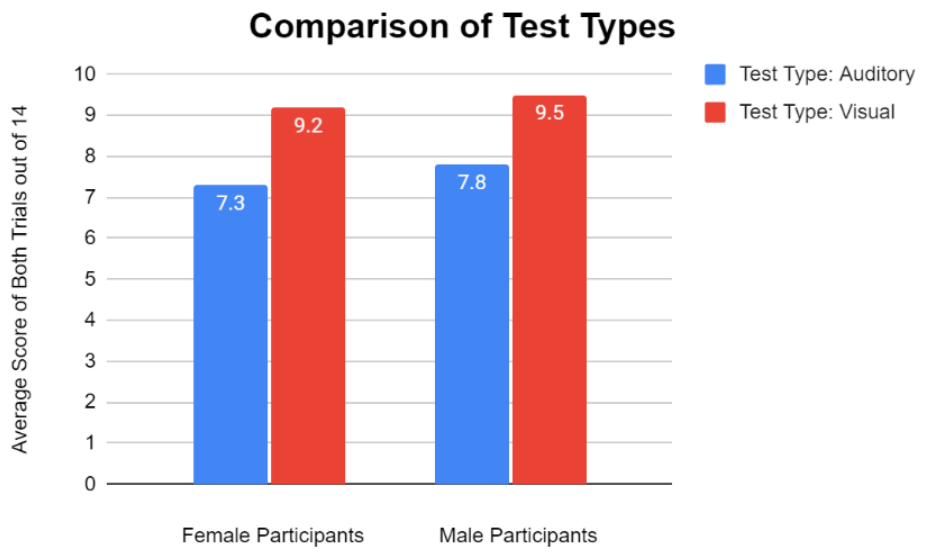

Figure 3. Comparison of Average Auditory and Visual Test Scores of Both Females and Males

\section{Discussion}

For future experiments, an analysis involving patients with Alzheimer's Disease could be an extension of this research. The purpose of the experiment would be to use it as an assessment of an individual's ability to retain memory, and conversely, not retain memory. An application of this experiment could be to test patients with memory loss and calculate their average score. The next step would be to test adults of the same age that do not have memory loss as a source of comparison. The results could be compiled, compared and analyzed, differentiating between the two categorized groups of participants. If, after repeated trials, their score is distinctly lower than the average score of adults who do not suffer from memory loss, or if their score falls within the range of scores of patients who do suffer from memory loss, they can use this as an indicator that they are in fact losing memory strength. For example, if a patient is beginning to feel like they are experiencing memory loss and finds themselves forgetting things on a consistent basis, they can take the auditory and visual memory tests that were created through this experiment. The advantage of performing this experiment is that it could be used as an indicator to detect possible onset of Alzheimer's Disease.

Another possible extension of this study could be to explore uses of these testing exercises as a therapeutic strengthening strategy for patients. If an individual is aware that their memory is weakening, they can use the tests designed in this experiment to strengthen their memory. Specifically, individuals suffering from Alzheimer's disease, as well as other diseases that directly affect memory loss, would benefit from practicing these tests. Interestingly, people who suffer from severe amnesia continue to have their auditory sensory memory intact. Therefore, by employing these testing strategies, it would be therapeutic for their memory, enhancing their ability to store information. 
The best way to improve something is to practice it. Therefore, by exercising their memory, patients who suffer from memory loss may strengthen their memory. The tests designed in this experiment certainly challenge and force participants to use their memory as best as they can, thus helping patients strengthen their memory. The visual test that was coded also records the participant's scores and catalogs their best performance. Therefore, patients can strive to improve their highest earned score by continuing to take the tests repeatedly, which improves their memory at the same time. If patients who suffer from memory loss continue to practice the memory tests designed, they are actively exercising their memory, thus using the test as a strengthening therapeutic.

\section{Conclusion}

Through the analysis of the collected data, each participant, regardless of their gender, demonstrated an improvement in their second trials. More so, collectively, participants performed better on the visual test than the auditory test. Overall, males obtained higher scores than females in each test type and likewise, increased their test score on the second trial. According to this data, males have a stronger visual and auditory memory than females. In conclusion, this experiment does not support the initial hypothesis. The initial hypothesis stated that auditory memory is stronger than visual memory, and that females have an increased capability in both the auditory and visual memory over males. Even though recent research indicates that recognition memory for sound is consistently stronger than recognition memory for images (Cohen et al., 2009), the experimental results support the opposing claim that visual memory is actually stronger than auditory memory in both males and females, and that males possess a stronger capability in both auditory and visual memory.

\section{Acknowledgements}

I would like to thank my school, North Shore Hebrew Academy High School, for allowing me the opportunity to conduct this research. Thank you to all the students who participated in this project and to their parents for allowing them to do so. Most of all, thank you to my advisor, Ms. McCarthy, for your support and help through this experience.

\section{References}

Archakov, D., DeWitt, I., Kusmierek, P., Ortiz-Rios, M., Cameron, D., Cui, D., Morin, E., VanMeter, J., Sams, M., Jaaskelainen, I., \& Rauschecker, J. (2020). Auditory Representation of Learned Sound Sequences in Motor Regions of the Macaque Brain. Proceedings of the National Academy of Sciences of the United States of America, 117(26): 15242-15252.

Barton, B. \& Brewer, A. (2013). Visual Working Memory in Human Cortex. Psychology (Irvine), 4(8): 655-662.

Brady, T., Konkle, T. \& Alvarez, G. (2011). A Review of Visual Memory Capacity: Beyond Individual Items and Towards Structured Representations. J Vis, 11(5): 4. https://www.ncbi.nlm.nih.gov/pmc/articles/PMC3405498/

Cohen, M., Horowitz, T. \& Wolfe, J. (2009). Auditory Recognition Memory is Inferior to Visual Recognition Memory. Proceedings of the National Academy of Science of the United States of America, 106(14): 6008-6010.

Ferguson, Melanie \& Henshaw, Helen (2015). Auditory Training Can Improve Working Memory, Attention, and Communication in Adverse Conditions for Adults with Hearing Loss. Frontiers in Psychology, 6: 556.

https://www.ncbi.nlm.nih.gov/pmc/articles/PMC4447061/ 
Friedman, G., Johnson, L., \& Williams, Z. (2018). Long-Term Visual Memory and Its Role in Learning Suppression. Frontiers in Psychology, 9: 1896. https:/www.ncbi.nlm.nih.gov/pmc/articles/PMC6194155/

Gloeds, M. \& Gregg, M. (2019). The Fidelity of Visual and Auditory Memory. Psychon Bull Rev 26, 1325-1332. https://doi.org/10.3758/s13423-019-01597-7

Lu, Z., Neuse, J., Madigan, S. \& Dosher, B. (2005). Fast Decay of Iconic Memory in Observers with Mild Cognitive Impairments. Proceedings of the National Academy of Science of the United States of America, 102(5): 17971802.

Nees, Michael (2016). Have We Forgotten Auditory Sensory Memory? Retention Intervals in Studies of Nonverbal Auditory Working Memory. Frontiers in Psychology, 7: 1892. https://www.ncbi.nlm.nih.gov/pmc/articles/PMC5133429/

Nishihara, M., Inui, K., Morita, T., Kodaira, M., Mochizuki, H., Otsuru, N., Motomura, E., Ushida, T. \& Kakigi, R. (2014). Echoic Memory: Investigation of Its Temporal Resolution by Auditory Offset Cortical Responses. PLOS ONE, 9(8). https://www.ncbi.nlm.nih.gov/pmc/articles/PMC4149571/

Plakke, B. \& Romanski, L. (2014). Auditory Connections and Functions of Prefrontal Cortex. Frontiers in Neuroscience, 8:199. https://www.ncbi.nlm.nih.gov/pmc/articles/PMC4107948/ 